\title{
LA POLÍTICA SOVIÉTICA Y CUBANA HACIA NICARAGUA: 1979-1989
}

\author{
Edmé Domínguez Reyes \\ Department of Peace E Development \\ University of Göteborg. Sweden
}

\section{Resum}

L'article està orientat vers la descripció i l'explicació de les polítiques soviètica $i$ cubana respecte a Nicaragua, des de 1979. Cuba proporciona suport - principalment en recursos bumans - a la revolució nicaragüenca des del primer moment, i es planteja com a objectiu fonamental la supervivència de la revolució. L'URSS, al contrari, mostra inicialment poc interès vers el procés nicaragüenc, dins d'una política general cap a Amèrica Llatina essencialment pragmàtica $i$ guiada per interessos propis en el marc de la confrontació bipolar global. A partir del 1981, $i$ a mesura que creix la pressió nord-americana, comença una nova època de relacions que anirà intensificant l'ajuda - principalment econòmica-de l'URSS i el CAME vers Nicaragua.

Resumen

El artículo está orientado hacia la descripción y explicación de las políticas soviética y cubana sobre Nicaragua desde 1979. Cuba suministra apoyo - principalmente en recursos bumanos - a la revolución nicaragüense desde el primer momento, planteándose como objetivo fundamental la supervivencia de la revolución. La URSS, por el contrario, muestra inicialmente poco interés hacia el proceso nicaragüense, en una politica general hacia Latinoamérica esencialmente pragmática y guiada por los intereses propios en el marco de la confrontación bipolar global. A partir de 1981, y a medida que crece la presión norteamericana, empieza una nueva época de las relaciones y se irá intensificando la ayuda -principalmente económicade la URSS y el CAME bacia Nicaragua.

Abstract

The article seeks to describe and explain the soviet and cuban policies towards Nicaragua from 1979 on. Cuba gave support - mainly in buman resources - to the Nicaraguan revolution from the first moment. The main purpose of this support was the survivance of the revolution. The USSR on the contrary showed first a weak interest in the Nicaraguan process, in the context of its general policies towards South America which is more pragmatic. Soviet policies expressed the USSR's interest in the global bipolar confrontation. From 1981 on, since the USA's pressure increased, a new age of relations began and there was an increasement in the USSR and CAME's support - specially economic- to Nicaragua. 
«Papers»: Revista de Sociologia

\section{INTRODUCCIÓN}

Cuba y la Unión Soviética representan dos estilos distintos de política en cuanto a su relación con el Tercer Mundo. Esta diferencia refleja su propia experiencia histórica pero también su lugar en la comunidad internacional e incluso sus objetivos ideológicos.

Desde los primeros años en el poder, el liderazgo revolucionario cubano intentó alentar el proceso revolucionario en otros países de Latinoamérica y el Caribe. El fracaso de estos intentos revolucionarios en otra parte del continente forzó a la dirección cubana a revisar y modificar su política externa en un sentido más pragmático. Durante los años 70 tal política se hizo más flexible ideológicamente, aceptando la posibilidad de otras formas para que la revolución obtuviera el poder (a través de los «militares progresivos» y de la «vía pacífica» chilena), y más pragmática, al reconocer la necesidad de restablecer relaciones con «todo tipo de gobiernos», sobre la base de un mutuo respeto en sus asuntos internos. Sin embargo, subsistió la "chispa revolucionaria», $\tan$ fuerte durante los años 70 . Reapareció en nuevas formas y países, como el apoyo militar dado al gobierno del MPLA en Angola.

Incluso en Latinoamérica, a pesar del nuevo pragmatismo y flexibilidad ideológica, Cuba mantuvo viva la «chispa revolucionaria», en forma de contactos con ciertos grupos revolucionarios sobrevivientes del período revolucionario de los años 70. Esta vez, sin embargo, no fue una cuestión de organización de «expediciones revolucionarias» guiadas por cubanos voluntarios. La asistencia cubana consistió en ayudar a aquellos grupos revolucionarios que estaban bien arraigados en su medio, especialmente en Centroamérica, de una manera no sólo logística sino también, y sobre todo, política, esto es, unificando facciones. Una vez en el poder, los revolucionarios contarían con el apoyo cubano en forma de ayuda material aunque, como veremos, este apoyo vendría junto con asesorías muy pragmáticas.

Para la Unión Soviética, esta época de apoyo revolucionario a grupos clandestinos del Tercer Mundo terminó durante la Segunda Guerra Mundial, junto con la desaparición del Comintern. Más aún, en cuanto a Latinoamérica, la Unión Soviética siempre ha sido más bien escéptica sobre sus posibilidades revolucionarias, dada su proximidad con los Estados Unidos. Cuba aparece como la excepción que confirma la regla. Su existencia como país socialista tiene mucho que ver con las circunstancias que rodearon a su revolución y la presencia de Khrushchev dentro de la dirección soviética. La posibilidad de que una experiencia de este tipo se pudiera repetir, la aceptación de otro país latinoamericano como miembro de la comunidad socialista, es hoy en día impensable. La política soviética respecto a Latinoamérica desde finales de los años 70 es muy pragmática, reflejando 
totalmente los intereses del Estado Soviético a los cuales se han adaptado perfectamente los partidos comunistas latinoamericanos. El caso de Granada y el de Nicaragua, aunque en cierta forma excepcionales, también son un ejemplo de tal pragmatismo, referente a los límites de la ayuda y la atención dada a tales países.

Este trabajo intentará centrarse en la política cubana y la soviética respecto a Nicaragua, situándolas dentro de su política general hacia Centroamérica. Deseamos presentar un enfoque comparativo de estas políticas con el fin de descubrir su complementariedad, coincidencias y contradicciones. Esto podría considerarse como un caso de estudio de las diferencias y similitudes entre las políticas soviética y cubana hacia el Tercer Mundo, aunque somos conscientes del peligro de extrapolar esta experiencia a otros contextos como el de África o Asia.

\section{LA UNIÓN SOVIÉTICA Y CUBA EN CENTROAMÉRICA}

Centroamérica es un área en la que la Unión Soviética y Cuba tienen intereses muy distintos. Para Cuba esta región es sumamente importante, por ser éste su medio natural. Cuba comparte con los países del área rasgos culturales similares, el mismo idioma, la misma historia (referente al colonialismo español y a las intervenciones y neocolonialismo norteamericano) y condiciones socioeconómicas semejantes.

Desde los comienzos de la revolución cubana, los dirigentes cubanos conocían las posibilidades revolucionarias de esta área. Los líderes cubanos recordaban la experiencia histórica de la insurrección de El Salvador en 1932, de la lucha de Sandino contra la ocupación norteamericana a principios de los años 30 y la experiencia reformista de Arbenz en Guatemala y su trágico resultado en 1954. Esto condujo a los dirigentes cubanos, ya en 1959, a alentar «expediciones revolucionarias» a países como Panamá, Nicaragua y la República Dominicana ${ }^{1}$. El fracaso de tales experiencias no impidió que varios grupos «castristas» se organizaran en algunos de estos países. Ciertos grupos sobrevivieron a la ola de represión que les hizo frente y pudieron mantener contactos con La Habana, aunque los intereses de Cuba en la revolución estaban centrados en Sudamérica desde mediados de los años 70.

La flexibilidad ideológica y el pragmatismo de Cuba durante los años 70 le permitieron acoger y aprobar el movimiento de Torrijos como «patriótico,

1. Véase: José Moreno, A. Lardas, «Integrating International Revolution and Detente: the Cuban Case» (Latin American Perspectives, vol. VI, núm. 2 primavera 1979), p. 41. 
«Papers»: Revista de Sociologia

popular y antiimperialista». Más aún, los Tratados del Canal firmados en 1977 entre Panamá y los Estados Unidos fueron particularmente elogiados por Fidel Castro como otra victoria latinoamericana contra el imperialismo. Sin embargo, para estas fechas, se estaban forjando nuevos movimientos revolucionarios y Cuba, aunque con sorpresa, también los acogería.

Para la Unión Soviética, Centroamérica nunca ha sido una de sus prioridades geográficas. Los dirigentes soviéticos no se han interesado en esta región desde el punto de vista económico ni político, aunque ciertos intereses geopolíticos pudieran haber jugado algún papel en el caso de Cuba. Más aún, al igual que con el resto de Latinoamérica, la Unión Soviética se mostraba muy escéptica en cuanto a las posibilidades revolucionarias en Centroamérica hasta 1979. Los países en esta área no eran considerados por la Unión Soviética como aptos para involucrarse en ninguna «construcción socialista», dado su bajo nivel de desarrollo, similar al de algunos países asiáticos o africanos, no siendo éste el caso, de acuerdo con observadores soviéticos, de algunos países latinoamericanos (como Argentina, Brasil o México), cuyo grado de industrialización era uno de los más altos del Tercer Mundo. Centroamérica también era considerada por los especialistas soviéticos como perteneciente al «grupo más reaccionario y pro-imperialista» del continente con dictaduras militares clásicas ${ }^{2}$.

No había relaciones gubernamentales entre la URSS y Centroamérica excepto con Guatemala, Costa Rica y la República Dominicana, que habían reconocido a la Unión Soviética al final de la Segunda Guerra Mundial. Esto no se debía tanto a la Unión Soviética como al resultado de la influencia norteamericana, particularmente fuerte en estos países. Por su parte, los soviéticos estaban preparados para considerar cualquier intento de acercamiento, como en el caso de Costa Rica en 1977, dada su escasa fe en cualquier tipo de cambio revolucionario en la región y su preferencia por «cambios pacíficos», como la experiencia chilena de 1973.

La victoria sandinista sobre Somoza llegó como un suceso inesperado para la Unión Soviética, aunque no tanto para Cuba. Como hemos mencionado anteriormente, Cuba había mantenido contactos con grupos como el Frente Sandinista de Liberación Nacional (FSLN) desde su fundación en 1962. El líder, Carlos Fonseca Amador, y algunos otros miembros de este grupo habían sido entrenados en Cuba y esta relación continuó incluso después del asesinato de Fonseca Amador en 1976. En 1977, Cuba comenzó a darse cuenta del nuevo dinamismo tomado por el FSLN en Nicaragua y empezó a organizar acciones de solidaridad. Sin embargo, la principal contri-

2. Véase: Gonionskij, "Amerique latine: la lutte pour la deuxieme liberation" (La Vie Internationale, núm. 3, 1972), pp. 42-48. 
bución de Cuba a la lucha nicaragüense no se dio en términos de pertrechos y entrenamiento, sino que fue política ${ }^{3}$. Los dirigentes cubanos mediaron en 1978 para lograr la unificación de las tres facciones en las que se había dividido el FSLN desde 1973. Esto permitió que las actividades de la guerrilla en el campo se coordinaran con aquéllas de las ciudades (en los barrios, fábricas y entre la clase media) para lanzar su ofensiva final en marzo de 1979. La victoria del New Jewel Movement (NJM) en Granada, casi al mismo tiempo, parecía anunciar una nueva época revolucionaria en esta región. Las esperanzas y estrategias cubanas, la unidad de las fuerzas de izquierda y la lucha armada finalmente habían triunfado 4 .

Estos hechos, junto con la situación revolucionaria de El Salvador, modificaron la visión soviética con respecto a Centroamérica y estimularon la discusión sobre la viabilidad de la lucha armada dentro del área. Los análisis soviéticos, que reflejaban sentimientos mixtos de entusiasmo y preocupación, se enfocaron sobre tres puntos durante los primeros años: 1) la validez de la lucha armada para otros países de Centroamérica, 2) la estrategia de alianza entre las diversas fuerzas de la izquierda y 3 ) las tareas de reconstrucción después de la toma del poder 5 .

Dado el enorme entusiasmo que rodeaba a la revolución nicaragüense, la mayoría de los especialistas soviéticos aceptaron la inevitabilidad de la lucha armada en Centroamérica, a pesar de su anterior oposición en cuanto a Latinoamérica. Algunos especialistas como S. Mikoyan y Kiva Majdanik respaldaron especialmente esta forma de lucha ${ }^{6}$. Pero en Costa Rica y Honduras, en donde las condiciones todavía no estaban maduras, se creyó necesario combinar la lucha armada con las acciones políticas. Se le dio un énfasis especial a la unificación de la izquierda, aunque existía el desacuerdo

3. Costa Rica, Panamá y Venezuela contribuyeron mucho más que Cuba, en términos de pertrechos materiales y apoyo logístico, a la victoria del FSLN. A Cuba le preocupaba la vulnerabilidad de Nicaragua si los Estados Unidos llegaban a sospechar un involucramiento cubano importante. Véase W. M. Leogrande, «Cuba and Nicaragua» (Caribbean Review, invierno 1980), p. 12.

4. Durante su discurso tradicional a la nación del 26 de julio, Fidel Castro recalcó que los sucesos en Nicaragua y Granada habían probado que la lucha armada era el único camino para lograr una revolución en Latinoamérica. También subrayó que aunque habían otros gobiernos progresistas en la región, sólo Nicaragua, Granada y Cuba se habían liberado completamente del yugo imperialista. (Véase: Granma, julio 27,1980 ).

5. Véase la discusión organizada por el Instituto de Moscú de Latinoamérica: «Nicaragua: experiencia de una revolución victoriosa» (América Latina, Moscú, núm. 3, 1980), pp. 33-116.

6. Ibid. Estas posiciones fueron confirmadas por nuestras entrevistas con estos especialistas durante la primavera de 1984. 
en cuanto al papel respectivo del partido comunista y de otros grupos en estas alianzas. Con respecto a la reconstrucción, surgieron dos posiciones. A. Shulgovskij e I. Zorina favorecieron un largo periodo de pluralismo político, economía mixta y diversificación; otros, como K. Maidanik y 'Tania Vorozheikina pensaron que las transformaciones «democráticas» necesarias deberían dar lugar rápidamente a un programa socialista ${ }^{7}$.

A nivel estatal, las primeras reacciones soviéticas tendieron a ser cautelosas. En 1979 la Unión Soviética enfrentó serios problemas económicos, el final de la distensión, problemas relacionados con la ratificación del Segundo Tratado de Limitación de Armas Estratégicas (SALT II) y las reacciones occidentales a la ocupación de Afganistán. Más aún, Moscú fue obligado a asumir nuevas cargas económicas como resultado de la guerra entre China y Vietnam y su propia invasión de Afganistán.

Cuba también enfrentó sus propios problemas en ese momento. 1979 y 1980 fueron años difíciles para la economía cubana, como resultado de las calamidades naturales, la disminución de los precios internacionales de sus principales productos de exportación y deficiencias de su propia organización económica. En el exterior, Cuba comenzó a tener problemas con países como Venezuela, Panamá, Costa Rica, Jamaica, Colombia y Perú. Entre las razones de estas fricciones figuraban los cambios de gobierno (muchos de estos nuevos gobiernos eran muy conservadores y recelaban de Cuba), un flujo de refugiados cubanos que intentaron salir de Cuba a través de las embajadas de algunos de estos países y finalmente, rivalidades de influencia entre algunos de estos gobiernos y Cuba con respecto a Nicaragua.

Simultáneamente, los esfuerzos de normalización entre Cuba y los Estados Unidos se detuvieron en 1980 como consecuencia de este nuevo período de polarización y la llegada de la administración de Reagan al gobierno de los Estados Unidos.

Una ventaja de Cuba en este período fue su popularidad internacional entre el Movimiento de los No Alineados (MNA). Cuba fue elegida presidenta del movimiento y, en 1979, la junta cumbre tuvo lugar en La Habana.

Dentro de este contexto, Cuba y la Unión Soviética comenzaron a desarrollar una política con respecto a Nicaragua, cuya amplitud y evolución no estaba clara en los primeros momentos.

7. «Nicaragua: Experiencia... Ibid. y S. Mikoyan «Sobre las particularidades de la revolución en Nicaragua» (América Latina, núm. 7, 1982), p. 41. 
Los primeros elogios de Fidel Castro a la revolución nicaragüense subrayaron el hecho de que esta revolución era distinta de la cubana en muchos aspectos. Había sido realizada por fuerzas revolucionarias en alianza con la clase media y la burguesía nacional y de esta manera tenía un carácter nacional que los líderes nicaragüenses «con la suficiente prudencia» deseaban preservar durante el periodo de reconstrucción ${ }^{8}$.

Tales ideas serían desarrolladas y repetidas varias veces por los líderes cubanos siempre que se refirieran a la experiencia nicaragüense. De acuerdo con el punto de vista cubano, Nicaragua no experimentó el mismo tipo de contradicciones de clase que Cuba, debido a que la burguesía nicaragüense había elegido el lado de los revolucionarios contra la dictadura de Somoza. Además, Somoza poseía propiedades suficientes con las que empezar, una vez nacionalizadas, las primeras reformas del programa sandinista. No había, por lo tanto, necesidad de afectar otras propiedades privadas, con tal de que los propietarios cooperaran en el esfuerzo de reconstrucción. Esto dio a los sandinistas la posibilidad de aplicar su programa de «economía mixta» y «pluralismo político» complementado por una «política de no alineación» a nivel externo, lo que les permitía obtener tanta ayuda como fuera posible de todo tipo de países. Sin embargo, la opinión cubana se preocupaba por la seguridad de Nicaragua, lo que hizo que Fidel Castro pusiera énfasis en la necesidad de mantener a la gente armada para organizar un ejército adecuado con el fin de evitar la repetición de la experiencia guatemalteca de 1954 o la de Chile de $1973^{\circ}$.

Los dirigentes cubanos también tenían en mente otro tipo de diferencias entre la experiencia cubana y la nicaragüense. Nicaragua no era una isla como Cuba, tenía fronteras territoriales que defender y era por lo tanto muy vulnerable. Asimismo, Nicaragua no era, ni sería, un miembro de la comunidad socialista, según los cubanos entendían las intenciones soviéticas. En consecuencia, a pesar de las expresiones de solidaridad soviéticas, Nicaragua no podría contar con el mismo tipo de ayuda económica ni la misma protección militar con las que contó Cuba ${ }^{10}$.

La ayuda cubana a Nicaragua estaba, por lo tanto, concebida desde el principio como un complemento de la ayuda que Nicaragua pudiera obtener

8. Para ésta y las siguientes ideas véase: Fidel Castro Speeches (Pathfinder Press, Nueva York, 1981), pp. 291-338.

9. Véase el discurso de Fidel Castro del 26 de julio de 1980 en Ibid., p. 320.

10. Véase: Raul Soh, "La politique Exterieure de Cuba», (Amerique Latine, CETRAL, Paris, Janv-Mars 1984, pp. 68-72), p. 70. 
de otros países, incluyendo los Estados Unidos. Con el deseo de alentar esta asistencia pluralista, Fidel Castro incluso intentó apelar a una «campaña de estimulación" para ver qué país daba más ayuda a Nicaragua ${ }^{11}$.

No obstante, Cuba comenzó a mostrar su solidaridad con los sandinistas inmediatamente después de su victoria. El 25 de julio de 1979 un avión cubano cargado con 90 toneladas de comida y transportando 60 médicos y enfermeras aterrizó en Managua. Antes del fin del año, mil maestros, algunos técnicos en pesca $y$, presumiblemente, los primeros asesores militares cubanos llegaron a Nicaragua ${ }^{12}$.

Durante 1980 Cuba comenzó a formalizar su ayuda a través de la firma de acuerdos de asistencia económica, científica y técnica. El gobierno cubano también intentó ayudar a Nicaragua a cubrir sus necesidades más urgentes, donando, por ejemplo, un barco mercante de 10000 toneladas de capacidad, en enero de ese año. En abril, ya había 2000 cubanos en Nicaragua, la mayor parte profesores que intentaban ayudar en la campaña de alfabetización sandinista, aunque también había personal de sanidad y otro tipo de asesores ${ }^{13}$. En 1983 había ya 4000 cubanos en Nicaragua. De acuerdo con Fidel Castro la mitad de ellos eran maestros, 750 personal médico, 1000 trabajadores de la construcción y 200 asesores militares ${ }^{14}$.

Cuba ayudaba así a Nicaragua con el tipo de recursos que tenía, recursos humanos. El objetivo no era cubrir todas las necesidades nicaragüenses, sabían que no lo podían hacer, sino ayudar en las más inmediatas y asistir a los sandinistas para emprender reformas urgentes. En consecuencia, la ayuda cubana estuvo enfocada principalmente a los sectores educativo, de salud y de seguridad, aunque también se cubrieron algunas otras áreas económicas. A finales de 1980, Cuba fue el primer donador a Nicaragua con 27 millones de dólares, seguido por México (25.1 millones de dólares) y el Mercado Común Europeo (10.6 millones de dólares) ${ }^{15}$. De 1979 a 1982 Cuba había donado el equivalente a 286 millones de dólares, el $16.1 \%$ de la ayuda que Nicaragua había recibido durante esos años. Durante el mismo periodo, como grupo, Europa occidental había contribuido con el $47 \%$, Latinoamérica con el $28 \%$ y los países socialistas (dentro de los cuales Cuba era el segundo donador después de la Unión

11. Fidel Castro Speeches, Ibid., p. 308.

12. Ibid., p. 306.

13. W. M. Leogrande, «Cuba and Nicaragua», (Caribbean Review, invierno 1980), p. 14. También: US Department of state, «Cuban-Soviet Impact on the Western Hemisphere» (Current Policy, núm. 167, abril 17, 1980). $3,1983)$.

14. Fidel Castro, «Press Conference to American Journalists», (Granma, agosto

15. Naciones Unidas, «Assistence to Nicaragua», (Report of the SecretaryGeneral, Report A/37/135, United Nations, N.Y. Oct. 6, 1982), pp. 6-7. 
Soviética) con el $23.3 \%$ de la ayuda total ${ }^{16}$. En términos de créditos, hasta 1984 Nicaragua había recibido de Cuba 53.5 millones de dólares ${ }^{17}$. Estos no eran, sin embargo, todos los créditos concedidos por Cuba a Nicaragua hasta ese año. En 1981 Cuba acordó un paquete de 64 millones de dólares en créditos, a los que se añadieron 130 millones de dólares en 1982, para distintos proyectos sociales y para la construcción de un molino de azúcar. Los créditos para esta construcción se convirtieron finalmente en donación. De acuerdo con fuentes nicaragüenses, de 1979 a 1987 Nicaragua había recibido un total de 64.9 millones de dólares de Cuba (la mayor parte de los cuales fueron enviados en 1982: 49.9 millones). Esto colocaba a Cuba en el quinto lugar de los donadores a Nicaragua de los países socialistas ${ }^{18}$.

Con respecto al comercio, a partir de un nivel no existente, las exportaciones nicaragüenses a Cuba se incrementaron hasta llegar al $4.2 \%$ de sus exportaciones totales, mientras que sus importaciones de Cuba sólo eran ligeramente más altas: $5.2 \%$ en 1982 . Hacia 1986 las exportaciones de Nicaragua a Cuba tuvieron un monto de 1.4 millones de dólares, mientras que sus importaciones alcanzaron 33.6 millones de dólares en $1985^{19}$.

Todas estas cifras muestran que Cuba, aunque con recursos limitados, deseaba hacer lo mejor, mostrar su solidaridad con Nicaragua. Cuba realizó de hecho un esfuerzo notable, especialmente en 1982, incluso dentro del grupo de países socialistas, posiblemente debido a su proximidad geográfica y a sus afinidades económicas con Nicaragua, pero también debido a su enorme compromiso con la supervivencia de la revolución sandinista. Este compromiso fue particularmente notorio en su ayuda para organizar el ejército sandinista.

Aparentemente, el interés cubano por la seguridad nicaragüense hizo que la dirección cubana insistiera en tener asesores cubanos sólo en posiciones de asesoría claves en la organización del ejército sandinista, de la inteligencia militar y del ministerio del interior. Esto no fue demasiado bien aceptado por los gobiernos de Panamá, Venezuela y Costa Rica que también estaban inte-

16. Ibid. la mayor parte de esta ayuda no es necesariamente financiera sino de todos tipos. La cubana se dio principalmente en términos de recursos humanos.

17. Nicaragua, Ministerio de Cooperación Exterior, Reporte, 1985.

18. Ibid., también: Naciones Unidas, Asamblea General, «Assistance to Nicaragua», Special Economic and Disaster relief Assistance: Special Programs for Economic Assistance (Report of the Secretary General, A/38/218, oct. 14, 1983), pp. 3-5. Para las últimas cifras véase: Ministerio de Cooperación Externa, Contratación de Recursos Externos: 1979-1987. Managua, Nicaragua, 1988.

19. Nicaragua, Ministerio de Comercio Exterior, Comercio Exterior 1982-1983, Boletín Estadístico núm. 5, 1984. Para las cifras de 1985-1986 véase: IMF, Direction of Trade Statistics, Yearbook 1987, Washington, D.C. 
resados en participar en esta organización (para hacer contrapeso a la influencia cubana), pero a los que se les propuso otras áreas de asistencia ${ }^{20}$. Es posible que Cuba haya desconfiado de los vínculos de Panamá con los militares y la inteligencia norteamericanos (a través de las bases del canal). Estas fricciones tal vez contribuyeron a los problemas que Nicaragua y Cuba comenzarían a tener en sus relaciones con sus vecinos y al clima regional de polarización que se inició en 1980. Desde entonces ha habido mucha especulación en cuanto al número y a las actividades de los militares cubanos estacionados en Nicaragua. De acuerdo con Fidel Castro, en la víspera de la invasión norteamericana a Granada, Cuba sólo tenía 200 consejeros militares trabajando para el gobierno sandinista 21 .

Los temores cubanos referentes a las intenciones norteamericanas hacia Nicaragua después de esos acontecimientos hicieron que Cuba repatriara entre 1200 y 2000 de sus ciudadanos que trabajaban en Nicaragua (no se sabe cuántos de éstos eran militares) ${ }^{22}$. En esa misma ocasión Fidel Castro declaró que en el caso de una invasión similar a Nicaragua, Cuba se vería igualmente impotente que en el caso de Granada para ayudar a Nicaragua militarmente, pero que confiaba en la capacidad de los nicaragüenses para defenderse ${ }^{23}$. Más aún, a mediados de 1984, Castro desmintió el rumor de que Cuba había recibido Migs soviéticos que habrían sido enviados a Nicaragua 24 . Hacia 1986 seguía habiendo reportes no confirmados sobre la presencia de 2500 3000 consejeros militares cubanos en Nicaragua, situados en todos los niveles del ejército sandinista y del Ministerio del Interior ${ }^{25}$. No obstante, de acuerdo con las declaraciones oficiales, Cuba deseaba, y había propuesto de hecho, retirar todos los asesores si se alcanzaba un acuerdo regional en este senti$\mathrm{do}^{26}$.

El aliento de Cuba hacia Nicaragua, para seguir una política de diversificación de sus vínculos internacionales, hizo que Cuba respaldara la solicitud

20. «A revolutionary friendship turns sour» (Latin American Weekly Review, dic. 21,1979 ), p. 2.

21. Fidel Castro, «Press Conference to American...».

22. Véase: Keesing's Contemporary Archives, mayo 1984. También Newsweek, en. 2, 1984.

23. Fidel Castro, «Speech in the farewell act to the fallen heroes in Granada", (Embajada de Cuba, Estocolmo, Oficina de Prensa, nov. 14; 1983), p. 1.

24. Granma Weekly, 16 julio, 1984.

25. Véase: Latin American Regional Reports, en. 26, 1986. Sin embargo, estas cifras siguen siendo inferiores al número de «consejeros» que los Estados Unidos mantienen en Honduras y El Salvador, para no hablar de Panamá.

26. Casi todos los discursos o declaraciones de Fidel Castro referentes a este tema han insistido en este punto. Véase por ejemplo: «Entrevista a Fidel Castro por la Cadena norteamericana NBC» (Granma Sem. marzo 13, 1988). 
de Nicaragua para entrar en el Movimiento de los No Alineados en 1979. Siguiendo las iniciativas cubanas, el MNA ha demostrado ser un foro activo de apoyo a Nicaragua desde la evidencia de presiones norteamericanas sobre el gobierno sandinista en 1980. Cuba también respaldó los esfuerzos de Ni-. caragua por diversificar sus proveedores de armas. En este sentido, Nicaragua se acercó desde 1980 a la RFA, la RDA, Bélgica, España, México y Brasil. Con respecto a la ayuda económica y al apoyo político, el desarrollo de relaciones entre Nicaragua y la Internacional Socialista fue altamente elogiado y alentado por $\mathrm{Cuba}^{27}$. En general, la prensa y las declaraciones cubanas parecían apreciar los esfuerzos de Europa occidental por ayudar a Nicaragua, aunque daba la impresión de que estos esfuerzos se obstinaban en combatir la «influencia cubana» y ofrecer a los sandinistas un modelo de desarrollo socialdemócrata. Tal modelo, como hemos visto, coincidía con el que los mismos sandinistas y Cuba habían pensado como el mejor para Nicaragua, al menos durante un largo período de transición.

A pesar de sus promisorios comienzos, las relaciones nicaragüenses con la Internacional Socialista, y especialmente con su rama latinoamericana, se vieron deterioradas desde 1982-1983 como resultado de la atmósfera de polarización ${ }^{28}$. Esta polarización, y especialmente las presiones norteamericanas, hicieron que Nicaragua se acercara con más frecuencia a la comunidad socialista en busca de ayuda y apoyo, entrando en un proceso que se describirá en la parte de las relaciones soviéticas con Nicaragua.

Al mismo tiempo, al comenzar Nicaragua a depender cada vez más de la ayuda soviética y de la Europa del Este, el apoyo cubano, especialmente el material, empezó a ser más discreto. La ayuda cubana en cuanto a recursos humanos había estado dirigida a solucionar los primeros y más urgentes problemas con los que Nicaragua se enfrentaba en el primer momento después de la victoria revolucionaria. Desde 1981, como resultado de las acciones de las bandas contrarrevolucionarias en Nicaragua, fueron asesinados algunos maestros y técnicos cubanos. Como hemos observado, parte del personal cubano comenzó a regresar por las repercusiones de Granada. Cuba comenzó a preocuparse por la seguridad de su propio personal y por la vulnerabilidad de la revolución sandinista, por lo que su apoyo se hizo más importante en el nivel político. Sin embargo, su presencia en Nicaragua continuó en

27. Barthelemy Febrer, «Cuba dans la réalité Centre-Américaine et Caraïbe: quelques remarques sur les six dernières années" (La Documentation Française, Problèmes d'Amérique Latine, núm. 64, 1982. (N.D. 4663 4664), pp. 156-157.

28. La actitud de Venezuela se hizo especialmente hostil hacia Nicaragua y Willy Brandt tuvo que cancelar una reunión de la IS en Caracas en febrero de 1982. Véase: Ibid. p. 157. 
«Papers»: Revista de Sociologia

algunas áreas, aparentemente más como respuesta a las solicitudes nicaragüenses que debido a las iniciativas cubanas.

Esta evolución se vio complementada por el respaldo cubano a diversas iniciativas de paz para Centroamérica. Cuba apoyó la iniciativa mexicana presentada en un encuentro internacional de partidos políticos en Managua en 1982. Haciendo público este apoyo, Fidel Castro subrayó que «Latinoamérica no debe temer las armas cubanas...» ni ninguna otra falsa idea que el gobierno de los Estados Unidos quisiera difundir ${ }^{29}$. Cuba también dio una calurosa bienvenida a la iniciativa de Contadora (organizada por México, Panamá, Costa Rica y Venezuela) para poner fin, mediante negociaciones, a los conflictos en Centroamérica ${ }^{30}$. En esa misma línea, los Acuerdos de Esquipulas de agosto de 1987 fueron altamente elogiados por Fidel Castro. Después de los acuerdos, Daniel Ortega viajó a Cuba para discutirlos con los dirigentes cubanos. Ambos líderes acordaron expresar su deseo de firmar un tratado regional para la retirada de todas las tropas y asesores extranjeros de la región ${ }^{31}$. Por otra parte, analizando estos acuerdos, Ricardo Alarcón, viceministro cubano de relaciones exteriores, puso de relieve que este documento era el resultado del fracaso de la política estadounidense en el área, tanto en Nicaragua como en El Salvador, y del apoyo internacional a la negociación pacífica que las actividades del grupo de Contadora habían logrado despertar ${ }^{32}$. En 1988, Fidel Castro reiteró que la ayuda y presencia cubanas en Nicaragua ya no eran esenciales, que lo que Nicaragua necesitaba era la paz. De ahí el apoyo cubano a todo tipo de acuerdos y decisiones que los nicaragüenses tomaran ${ }^{33}$.

Aparentemente, los puntos de vista cubanos sobre el modelo nicaragüense no han cambiado mucho desde 1979. En 1982, cuando las presiones contra Nicaragua se incrementaron, los círculos cubanos internos pensaron que incluso si el modelo pluralista era el correcto para Nicaragua en ese momento, dadas las presiones internas y externas, «era preferible avanzar» (en la clase de transformaciones) «que retroceder porque, a pesar de todo, el socialismo sería posible» ${ }^{34}$. Estos puntos de vista estaban basados en los desacuerdos cubanos con respecto al comportamiento de los sectores privados nicaragüenses y las críticas provenientes de Europa Occidental sobre la actuación política y económica del gobierno sandinista.. Probablemente los

29. Granma, febr. 28, 1982.

30. Véase, por ejemplo, las declaraciones cubanas en Excélsior (México, marzo 31,1985$)$.

31. Granma Sem. ag. 23, 1987.

32. Granma Sem., dic. 6, 1987.

33. Véase: «Entrevista a Fidel Castro por... NBC». op. cit.

34. Granma Sem. dic. 6, 1987 y Latin America Regional Reports, oct. 29, 1987. 
cubanos también pensaban en su propia experiencia, en la que las presiones habían conducido a la radicalización de la revolución. Aparentemente, tales pensamientos no se tradujeron en consejos concretos a los dirigentes sandinistas para radicalizar su proceso. Por el contrario, hasta ahora la línea de prudencia cubana parece continuar inalterada.

Así pues, la política cubana hacia Nicaragua ha sido de apoyo y respeto incuestionables a todos niveles. La ayuda cubana hacia Nicaragua sólo es un aspecto de esta política, aunque esta ayuda haya jugado un papel importante para la supervivencia nicaragüense, especialmente en los primeros momentos. De hecho, en la actualidad la prensa cubana sigue remarcando algunos aspectos de esta ayuda, como la educación y la capacitación técnica dada a los estudiantes nicaragüenses en Cuba (1200 en 1987) o las 40.000 t de petróleo donado a Nicaragua durante 1987 , para ayudar a compensar parte de la cuota que los soviéticos querían disminuir (véase más adelante) ${ }^{35}$.

Más importante que su ayuda ha sido el respaldo cubano a los dirigentes sandinistas durante los períodos más difíciles. Este apoyo moral y político ha continuado sin ninguna crítica pública de los dirigentes cubanos contra el gobierno sandinista, como ha sido el caso de otros países que habían ayudado a Nicaragua en uno u otro momento (como Venezuela, Costa Rica o los países de Europa Occidental). Pudo haber fricciones entre los dos gobiernos (hubo rumores en ese sentido sobre críticas cubanas a los sandinistas por su falta de flexibilidad con respecto a las negociaciones con los Estados Unidos), pero éstas no se han utilizado como el tipo de presión que otros países han tratado de ejercer sobre el gobierno sandinista. Más aún, en la entrevista realizada por la NBC en 1988, Fidel Castro remarcó que Cuba respaldaba cualquier decisión que los sandinistas tomaran en cuanto a su forma de gobierno o sus relaciones exteriores ${ }^{36}$.

Finalmente, algo que debe subrayarse es la similitud de posiciones entre Cuba y Nicaragua con respecto al gobierno de Noriega en Panamá. Su defensa de este gobierno se debió principalmente a su posición antinorteamericana y sus sospechas de un involucramiento norteamericano en los asuntos panameños. Tanto el gobierno nicaragüense como el cubano estaban al tanto del oportunismo de Noriega (sabían que Noriega había colaborado con la CIA contra ellos en cierta época), pero su antiimperialismo era más fuerte que cualquier contradicción anterior con Noriega, como había sido el caso durante la guerra de las Malvinas. Sus sospechas se vieron confirmadas, como hemos visto, con la invasión norteamericana de Panamá.

35. Granma Sem., dic. 6, 1987 y Latin America Regional Reports, oct. 29, 1987. 36. «Entrevista a Fidel Castro por... NBC», op. cit. 


\section{URSS-NICARAGUA}

La Unión Soviética prácticamente no prestó atención a Nicaragua antes de 1979. De acuerdo con algunos eruditos soviéticos, Nicaragua apenas si habría sido candidata para transformaciones revolucionarias, debido a la debilidad de su clase trabajadora y a la fuerza de la dictadura de Somoza. No fue sino hasta agosto de 1978 que los soviéticos observaron la lucha sandinista con interés. No obstante, Moscú fue de los primeros en felicitar y reconocer al nuevo régimen sandinista.

Por su parte, Nicaragua consideró a la Unión Soviética como uno más de los países alternativos de Europa Oriental y Occidental, dentro de su política de diversificación y no alineación.

La política soviética hacia Nicaragua se divide en dos periodos: 1) un periodo de explotación de 1979 a 1981, durante el cual el papel de la URSS en la reconstrucción fue insignificante, comparado con el de Cuba o Latinoamérica como un todo, y 2) un periodo de compromiso creciente desde 1981 hasta la fecha, en que la ayuda soviética se ha vuelto más importante en vista del virtual bloqueo de los Estados Unidos y la declinación de la popularidad de Nicaragua entre algunos gobiernos latinoamericanos y europeos, y los socialdemócratas ${ }^{37}$.

Durante este primer periodo se dio el establecimiento de líneas de cooperación ideológica y política entre los sandinistas y el PCUS. La primera delegación partió hacia Moscú en marzo de 1980, donde firmó los primeros acuerdos sobre cooperación política, comercio y ayuda económica y técni$\mathrm{ca}^{38}$. Desde el punto de vista sandinista, la cooperación política probablemente era considerada útil para el establecimiento de futuras líneas de ayuda económica proveniente de los países socialistas si así se requería, y como parte del esfuerzo de los sandinistas por diversificar sus relaciones políticas ${ }^{39}$. Por su parte, los analistas soviéticos, como I. Bulichev, aunque reconocían al FSLN como la vanguardia de la revolución nicaragüense (que dio al FSLN el

37. Esta declinación se debió en parte a las presiones norteamericanas sobre los aliados europeos para que no continuaran ayudando a Nicaragua, y en parte a las expectativas occidentales no satisfechas, relativas al pluralismo político de Nicaragua. Vêase por ejemplo, Robert Mathews, «The limits of friendship: Nicaragua and the West» (en Nacla, Report of the Americas, mayo-junio 1985).

38. Véase: Sandino's Ideological Heritage, Progress edit., Moscú, 1980, p. 36. De acuerdo con el agregado comercial de Nicaragua en Moscú, se firmó un protocolo de comercio desde esta fecha. Dentro de este protocolo la Unión Soviética se comprometió a comprar 20.000 tons. de algodón, 5.000 tons. de café y 15.000 tons. de azúcar. (Entrevista, Moscú, junio 1984).

39. Véase: Patria Libre (Managua), núm. 10, en. 1981, pp. 32-33. 
carácter de un partido comunista, siendo el antiguo PSN prácticamente ignorado), intentaron subrayar la necesidad de una unidad nacional y un pluralismo económico como las características principales de la reconstrucción nicaragüense $\mathrm{e}^{40}$.

Como resultado de las crecientes tensiones con la administración de Reagan, el gobierno sandinista comenzó a solicitar ayuda militar y económica desde 1981. En ese año, se solicitaron los primeros tanques soviéticos y Moscú proporcionó créditos de hasta 73.2 millones de dólares que se usarían principalmente para la importación de bienes soviéticos ${ }^{41}$. Después de la visita de estado de Daniel Ortega a Moscú en mayo de 1982, Nicaragua recibió créditos soviéticos adicionales para proyectos hidroeléctricos a gran escala, diques secos en la costa del Pacífico y la construcción de una estación de tierra que se conectaría con el sistema Intersputnik. En ese año, la Unión Soviética se convirtió en el primer acreedor de Nicaragua, con 150 millones de dólares ${ }^{42}$.

La mayoría de los análisis soviéticos sobre Nicaragua, desarrollados por especialistas como Smirnova y Bulichev, continuaron haciendo hincapié en el carácter democrático de las transformaciones nicaragüenses -democráticorevolucionarias - pero remarcaron la necesidad de que los sandinistas estuvieran alerta y consolidaran su poder ${ }^{43}$.

Es importante observar que uniendo la ayuda recibida por Nicaragua proveniente de fuentes bilaterales durante este periodo (1979-1982), los países socialistas (la URSS, Cuba y Europa del Este) alcanzan el segundo lugar (460.1 millones de dólares), después de Latinoamérica (634 millones de dólares). Dentro de la ayuda de países socialistas, aparte de Cuba (54.5 millones de dólares), la RDA da 108 millones de dólares ${ }^{44}$.

40. Bulichev, «El fortalecimiento de la unidad nacional» (America Latina, núm. 7, 1981, pp. 40-56.

41. Véase: SIPRI Yearbook (Estocolmo, Institute for the Peace research), 1983, pp. 325-336. Incluida en la ayuda económica, 6.8 millones de dólares debían usarse para la apertura de tres escuelas de capacitación técnica y 16.6 millones de dólares para la instalación de dos centros de transmisión radial. Véase: United Nations, General Assembly, Report of Assistance to Nicaragua (...14 oct., 1983. A/38/218) pp. 4-6.

42. United Nations, General Assembly, Report of... Ibid. Una selección de los documentos firmados durante esta visita se incluyeron en Sandino's Ideological Heritage, Ibid.

43. Bulichev, op. cit., N. Smirnova, «Nicaragua: la revolución en marcha» (América Latina, núm. 6, jul. 1982), pp. 4-12. Véanse también los distintos artículos aparecidos en: Nicaragua: Glorioso Camino a la Victoria (Moscú, Ciencias Sociales Contemporáneas, Academia de Ciencias de la URSS, 1983).

44. United Nations, General Assembly, Report of... ibid. 
«Papers»: Revista de Sociologia

En 1983 y 1984 tanto Andropov como Chernenko continuaron la política iniciada por Brejnev. Las tensiones dentro y fuera de la región continuaron incrementándose. La invasión estadounidense a Granada provocó la inquietud soviética, llevando a la URSS a creer en la posibilidad de una intervención estadounidense en Nicaragua. Como resultado, las declaraciones soviéticas se hicieron más cautelosas. Aparentemente, el mensaje que las autoridades soviéticas querían dar a entender era que Nicaragua no era Cuba y que en caso de una agresión norteamericana, Nicaragua tendría que defenderse con sus propios medios ${ }^{45}$. Tal actitud de bajo perfil persistió incluso después de que un barco soviético fuera dañado por minas puestas por la Agencia Central de Inteligencia (CIA) en puertos nicaragüenses. Aparte de las violentas condenas a las acciones norteamericanas, las autoridades soviéticas sólo reafirmaron su respaldo moral a Nicaragua ${ }^{46}$. Aparentemente esto también significaba una cierta limitación en la provisión de armas soviéticas, como parece indicar la búsqueda sandinista de pertrechos militares en otros países (Europa Occidental, Libia e Irán, entre otros) $)^{47}$.

La URSS continuó proveyendo ayuda económica en forma tanto de créditos bilaterales como de proyectos de asistencia, estos últimos del Consejo de Asistencia Mutua Económica (CAME). En la primera mitad de 1984, la ayuda económica soviética se mantuvo entre los 100 y los 150 millones de dólares, lo que constituyó un incremento de aproximadamente el $25 \%$ sobre la ayuda soviética de $1983^{48}$. Hasta 1983 la Unión Soviética y Europa del Este habían proporcionado el $19.9 \%$ del crédito total recibido por Nicaragua desde 1979. Durante el mismo periodo Latinoamérica proveyó el $32.2 \%$ de estos créditos ${ }^{49}$.

En mayo de 1985 la administración de Reagan decidió aplicar un embargo comercial a Nicaragua. El comercio exterior de Nicaragua sufrió una seria desestabilización. Asimismo México, que se había convertido en uno de los principales proveedores de petróleo de Nicaragua redujo abruptamente sus

45. Véase: Communique issued after Daniel Oretega's visit to the USSR in March 1983. Pravda (26 marzo 1983).

46. Pravda (8 abril 1984).

47. Nicaragua había firmado un contrato con Francia para comprar cierto equipo de armas sofisticadas por 15 millones de dólares Debido a la presión directa norteamericana, el gobierno de Mitterrand decidió interrumpir la entrega, rompiendo el contrato. Véase: The Financial Times, marzo 31, 1982. También: Robert Mathews «The limits of Friendship: Nicaragua and the West» (en NACLA, Report of the Americas, Sandinista Foreign Policy, mayo-junio 1985), p. 30.

48. New York Times (28 marzo 1984).

49. «Reporte» (Banco Central de Nicaragua), 1984. 
entregas petroleras debido a su propia situación económica y a los problemas de Nicaragua para pagar sus deudas ${ }^{50}$.

Para enfrentarse con esta situación, el gobierno sandinista trató de intensificar su política de diversificación de vínculos externos. El presidente Ortega viajó dos veces a Moscú en 1985 (en marzo asistió al funeral de Chernenko y en mayo visitó otras capitales de Europa del este). En 1983 Nicaragua ingresó en el CAME como «miembro observador» (la misma posición que México e Irán recibieron en los años 70) y obtuvo apoyo financiero y técnico de este organismo para 12 proyectos diferentes (industrialización, irrigación, capacitación técnica para especialistas en distintas áreas, etc. $)^{51}$.

Dadas las pequeñas cantidades de ayuda económica y militar obtenidas por Nicaragua en otros países, los sandinistas solicitaron y obtuvieron ayuda de la URSS y de los países socialistas en cantidades crecientes. En 1985 la ayuda económica soviética estaba por encima de los 500 millones de dólares $y$, de acuerdo con fuentes norteamericanas, la ayuda militar había obtenido las mismas cantidades ${ }^{52}$. Asimismo, en 1985 la URSS proporcionaba a Nicaragua el $80-90 \%$ de sus requerimientos de petróleo ${ }^{53}$. Sin embargo, esto cambió drásticamente; en la primavera de 1987 la URSS anunció que reduciría sus contribuciones de petróleo, y del $80 \%$ de las necesidades nicaragüenses pasó sólo a suministrar el $40 \%$ en $1988^{54}$. La ayuda soviética total se calculó en 600 millones de dólares durante 1986. En enero de 1987 la Unión Soviética firmó acuerdos para la provisión de 300 millones de dólares para ese año en distintos proyectos de asistencia ${ }^{55}$.

Por otra parte, los intercambios comerciales entre Nicaragua y la URSS progresaron a un ritmo más lento. En 1981 Nicaragua exportó el $6.2 \%$ de sus exportaciones generales a la URSS e importó el $1.5 \%$ de sus importaciones

50. México había estado proveyendo no sólo a Nicaragua, sino a la mayoría de los países centroamericanos y algunos caribeños 160.000 barriles diarios de petróleo como parte del acuerdo hecho con Venezuela en 1982, el llamado "Pacto de San José». Los problemas propios de Venezuela también dificultaron la continuación de este acuerdo. Para más datos sobre esto, véase: Richard R. Fagen, Olga Pellicer, edit. The Future of Central America (Stanford University Press, 1983), pp. 119-133.

51. Véase Pravda (10 mayo 1985). Para los acuerdos del CAME véase: entrevista a Carlos Rafael Rodríguez (Cuba Internacional), núm. 8 1984. También: Yearbook of International Communist Affairs (YICA), 1986, p. 127.

52. YICA ibid. De acuerdo con fuentes nicaragüenses, los créditos soviéticos totales alcanzaron los 664.1 millones de dólares en 1985, véase la nota 18.

53. The Washington Post, mayo 21, 1985.

54. Véase: Newsweek (junio 22, 1987), p. 27.

55. Latin American Monitor, en., febr., 1987. 
totales $^{56}$. En 1983 se llegó al $1.6 \%$ y $8.2 \%$, respectivamente. De acuerdo con fuentes soviéticas, a finales de 1987 Nicaragua había exportado 13.2 millones de rublos a la URSS e importado aproximadamente 166.1 millones de rublos (un decremento importante respecto de los 226 millones de rublos que había importado en 1986) ${ }^{57}$. Con Europa del Este estos intercambios crecieron de una forma más rápida. Hacia 1983 Nicaragua exportó el $12.7 \%$ de sus exportaciones al CAME e importó el 16.6\% de sus importaciones. En 1984 las exportaciones declinaron hasta llegar al $6.1 \%$ y las importaciones se incrementaron hasta alcanzar un $26.5 \%$. En 1986 se calculó que la parte del CAME del comercio exterior de Nicaragua se elevaría al 35\%. Sin embargo, a principios de 1985 Nicaragua seguía comerciando con los Estados Unidos tanto como con Europa del Este ${ }^{58}$.

La estructura de comercio de estos intercambios sigue siendo muy tradicional. Nicaragua exporta principalmente mercancías agrícolas, café, algodón, semillas de ajonjolí e importa de Europa del Este (incluyendo la URSS) bienes de capital, manufacturas y productos de consumo y combustible. Las modalidades de pago son, sin embargo, más flexibles que las de occidente: algunas veces incluyen una opción de pago en moneda de poco cambio (acuerdos de trueque) o pago en productos producidos por instalaciones de fabricación ligera montadas por los países del CAME. Por ejemplo, las agroindustrias que Bulgaria financió se pagarán con productos nicaragüenses enlatados y procesados por estas industrias ${ }^{59}$. Por otra parte, estos países rara vez han ofrecido a Nicaragua precios preferenciales por sus productos ${ }^{60}$.

Los créditos dados por los países socialistas a Nicaragua se incrementaron de 102 millones de dólares en 1980 hasta un máximo de 252.9 millones de dólares en 1982 y 146 millones en 1983 . Desde 1979 y hasta 1984 Nicaragua había recibido 262.2 millones de dólares de la URSS y 606.1 millones del CAME. Durante el mismo periodo Latinoamérica había pro-

56. Ruben Berríos, Marc Edelman, «Diversifying Dependence: Nicaragua's new economic links, with the Socialist Countries", The Journal of Communist Studies, (vol. 2, núm. 1, marzo, 1986), pp. 31-48.

57. Comercio Exterior, Moscú (núm. 12, 1987).

58. Ibid. De acuerdo con el IMF Yearbook 1987, en 1983 Nicaragua exportó sólo el $5.6 \%$ de sus exportaciones totales al CAME, mientras que importó el $12.0 \%$ de sus importaciones totales. Para 1985 los porcentajes fueron $8.3 \%$ y $14 \%$, respectivamente. Véase IMF... op. cit.

59. Para acuerdos de comercio véase: CEPAL, Relaciones económicas de América Latina con los paises miembros del Consejo de Asistencia Mutua Económica (Santiago: estudios e informes de la CEPAL, núm. 12, 1982), p. 42. También Rubén Berríos... Ibid., p. 42.

60. $12 \mathrm{Ibid}$, p. 42. 
porcionado 762.5 millones de dólares a Nicaragua ${ }^{61}$. Para 1984 la mayoría de los créditos obtenidos por Nicaragua bilateralmente provenían de los países socialistas. De 1979 a 1987 estos países habían proporcionado 2272 millones de dólares en créditos, de los cuales 1394 provenían de la Unión Soviética ${ }^{62}$.

El caso de Nicaragua se asemeja mucho al de Cuba en los años 70. La hostilidad norteamericana es casi la misma (guerra secreta, embargo), pero la respuesta soviética y la propia evolución interna de Nicaragua son distintas. Está bastante claro que la Unión Soviética está dispuesta a ayudar a Nicaragua a sobrevivir, especialmente dadas ciertas afinidades políticas e ideológicas y su objetivo general de cultivar el antinorteamericanismo dentro de Latinoamérica, pero no está preparada para darle la bienvenida a la comunidad socialista y para comprometerse en su defensa. Últimamente esta actitud se ha vuelto más evidente con el anuncio soviético de la reducción radical de provisiones de petróleo a Nicaragua en la primera mitad de 1987, con la falta de incremento importante de la ayuda soviética después de la visita de Ortega a Moscú en octubre de 1987 y con la aprobación soviética del nuevo acuerdo de Paz Centroamericano firmado en agosto de $1987^{63}$. Aunque el gobierno de la Unión Soviética restableció sus entregas normales de petróleo en agosto de 1987 y se comprometió a cubrir como siempre las necesidades nicaragüenses para el resto del año, está claro que Moscú está intentando bajar su perfil en Nicaragua ${ }^{64}$.

En 1989 Moscú anunció que había iniciado las disminución de asistencia militar a Nicaragua desde finales de 1988. La visita de Shevardnandze a Managua (la primera de un funcionario soviético de tan alto rango) en octubre de 1988 confirmó esta reducción de entregas de armas y anunció una nueva época de cooperación económica en un contexto de paz (abierto por acuerdos centroamericanos). De acuerdo con Shevardnaze, la Unión Soviética había enviado hasta aquel momento 1400 millones de rublos en bienes no militares, 300 millones de rublos en donaciones y había colaborado en

61. United Nations, General Assembly, op. cit., Berríos, Edelman, op. cit. y Nicaragua, Ministerio de Cooperación Exterior, 1985.

62. Ministerio de Cooperación Exterior. Ibid, p. 9.

63. En general la política exterior de Gorbachev ha intentado dar una baja prioridad al Tercer Mundo. En su Reporte del $27^{\circ}$ Congreso de febrero de 1986, Gorbachev puso enfásis en la necesidad de encontrar iniciativas para "soluciones colectivas" referentes a áreas regionales en conflicto. Véase también el artículo de Robert Legvold en el International Herald Tribune (nov. 17, 1987). Para la actitud soviética hacia el acuerdo de Paz, véase Pravda e Izvestia, 8, 9, 10 ago. 1987. También: Caribbean Insight, nov. 1987.

64. Véase: Caribbean Insight, jul., sept., oct. 1987. 
40 proyectos industriales o agrícolas conjuntos en Nicaragua ${ }^{65}$. El mensaje soviético a través de esta visita pareció ser un estímulo para los esfuerzos de paz regional (Shevardnaze declaró el deseo soviético de restablecer relaciones con todos los países de Centroamérica) y para que el régimen sandinista continuara sus esfuerzos de democratización y desmilitarización. Parece que las intenciones soviéticas llegan al punto de proponer a los sandinistas cierto tipo de mediación con los Estados Unidos, con el fin de obtener una solución negociada a los conflictos que afligen esta región. Esto sigue el patrón abierto por Gorbachev relativo a su política hacia los conflictos regionales.

Por otra parte, las prioridades soviéticas, el costo económico, las razones de seguridad y el propio plan de reformas de Gorbachev, junto con un deseo de mejorar las relaciones soviéticas con los Estados Unidos, entre otros, pueden explicar esta actitud. Nicaragua, por su parte, está comprometida con un estilo de desarrollo de economía mixta, en la que el sector privado sigue contando con el $60 \%$ de su PNB, y con una política de no alineación. Los reclamos sandinistas socialistas están más relacionados con su programa a largo plazo que con su futuro presente o inmediato. En estas condiciones, la perspectivas de una cooperación soviético-nicaragüense dependen mucho de las acciones norteamericanas, pero en cualquier caso es altamente improbable que tal cooperación evolucione de la misma manera que lo hizo la soviéticacubana.

\section{CONCLUSIONES}

La principal preocupación de Cuba con respecto a Nicaragua ha sido su supervivencia. Por lo tanto, aparte de su ayuda directa y apoyo político, es probable que Cuba haya jugado un importante papel como mediador con Europa del Este. De cualquier forma, tal interés por la supervivencia de la revolución nicaragüense ha vuelto a los líderes cubanos muy consistentes en su respaldo a cualquier acuerdo pacífico que pudiera aliviar la carga nicaragüense y le permitiera lograr si no su desarrollo revolucionario, al menos un desarrollo democrático.

La Unión Soviética también ha estado interesada en la supervivencia de la revolución sandinista. Su ayuda a Nicaragua ha sido más importante en cuanto a las cuestiones financieras y materiales que la dada por Cuba, pero su naturaleza ha sido diferente. La ayuda cubana, principalmente mediante recursos humanos, ha sido enviada a aquellas áreas sociales que necesitan el

65. Tass, oct. 5 y Pravda, oct. 6, 1989. 
tipo de cambios que una revolución popular debe realizar primero, por ejemplo, la educación y la salud. La ayuda soviética se ha enfocado a proyectos de desarrollo y otras áreas económicas. Sin embargo, ambos tipos de ayuda se complementan entre sí, lo que es particularmente evidente en el sector militar. La Unión Soviética ha proporcionado armas y equipo; los cubanos, asesores (aunque también hay asesores del la Europa del Este en este sector). Puede haber fricciones entre Cuba y los soviéticos con respecto a la cantidad de ayuda y otras materias, pero ambos han acordado que Nicaragua no puede esperar demasiado de la comunidad socialista, en todo caso no el mismo trato que Cuba.

Tanto Cuba como la Unión Soviética han respetado y alentado el programa nicaragüense de pluralismo político y economía mixta, y especialmente su objetivo de diversificación de vínculos externos. Ideológicamente pueden justificar este estímulo sobre la base de que sólo se trata de un periodo de transición, aunque, dadas las condiciones de Nicaragua, su situación geográfica y la actual orientación de la Perestroika, parece inevitable un desarrollo capitalista, al menos durante largo tiempo.

Los intereses cubanos y soviéticos son diferentes. La Unión Soviética es una superpotencia que hoy día está más preocupada por su relación con la otra superpotencia y sus propios problemas internos que por añadir un nuevo y oneroso miembro a una comunidad socialista que ya está en vías de desaparecer. Por lo tanto, el apoyo soviético a un país como Nicaragua tiene que ser limitado, como siempre lo ha sido, e incluso si se ha visto incrementado debido a las peticiones urgentes de Nicaragua, está en proceso de disminución, como parece indicar la visita de Shervardnaze. El interés de Cuba es preservar una Nicaragua democrática, si no revolucionaria, como un aliado seguro en la región, en vez de asistir a otra repetición de la experiencia chilena, especialmente después de la invasión norteamericana a Panamá.

Aunque con intereses diferentes, Cuba y la Unión Soviética han logrado complementar sus políticas hacia Nicaragua. El respeto, la flexibilidad y los compromisos limitados han sido las palabras clave. 